\title{
Periodically collapsing Evans bubbles and stock-price volatility
}

\author{
Benedikt Rotermann und Bernd Wilfling ${ }^{\dagger}$
}

$28 / 2013$

${ }^{\dagger}$ Department of Economics, University of Münster, Germany 


\title{
Periodically collapsing Evans bubbles and stock-price volatility
}

\author{
Benedikt Rotermann, Bernd Wilfling \\ Westfälische Wilhelms-Universität Münster, Department of Economics, Am \\ Stadtgraben 9, 48143 Münster, Germany
}

(Date of this version: November 26, 2013)

\begin{abstract}
This paper analyzes conditional stock-price volatility within in present-value framework including (rational) periodically collapsing bubbles as introduced by Evans (1991). To this end, we derive an analytically closed-form volatility formula of the stock price. The formula establishes a direct link between the bubble component and stock-price volatility. Using a Bayesian Monte-Carlo estimation technique (the particle filter), we demonstrate how to fit the parametric volatility equation to stock-market data.
\end{abstract}

JEL classification: $\mathrm{C} 1, \mathrm{G} 1$.

Keywords: Present value model, Evans bubbles, conditional volatility, particle filter estimation. 


\section{Introduction}

Several authors argue that the frequently observed excessive volatility in stock prices may be attributed to the presence of speculative bubbles. Blanchard and Watson (1982) and Flood and Hodrick (1986), inter alia, demonstrate in a theoretical framework that bubble components potentially generate excessive volatility. Besides the many articles discussing theoretical aspects of speculative bubbles and econometric techniques for their detection, there is a second strand of literature linking financial crises and/or bubbly periods to stock-price volatility. Two important pieces of research are Brunnermeier and Oehmke (2013) and the so-called Minsky model (as described in Kindleberger and Aliber, 2005, pp. 24-37) according to which financial crises and/or bubbly periods are characterized by different phases of stock-price volatility. During the early stages of a bubbly period stock-price volatility appears to be low whereas towards the end of the bubble and its burst stock-price volatility is typically high.

In this paper we consider the existence of periodically collapsing bubbles as proposed by Evans (1991) in the well-known present-value model and theoretically analyze conditional stock-price volatility within this framework. Using a sequential Bayesian Monte-Carlo technique, we fit our theoretical model equations to an artificial dataset to gain further insights into stock-price volatility dynamics during bubbly periods. Our analysis has two major findings. First, we show that our rational bubble specification entails excess stock-price volatility. Second, we find that the dynamic structure of this volatility dynamics accords with the phases of low and high volatility as proposed by Brunnermeier and Oehmke (2013) and the Minsky model.

\section{Present-value model and the Evans bubble}

In the linear present-value model with rational expectations the price of a stock at date $t, P_{t}$, is given by the Euler equation

$$
P_{t}=\frac{1}{1+r}\left[E_{t}\left(P_{t+1}\right)+E_{t}\left(D_{t+1}\right)\right],
$$


where $D_{t+1}$ is the stock dividend payment between $t$ and $t+1 . E_{t}(\cdot)$ denotes the conditional expectation operator based on all information available to market participant as of date $t . r$ is the required rate of return that is just sufficient to compensate investors for the inherent riskiness of the stock (see Campbell et al., 1997; Cuthbertson and Nitzsche, 2004). To solve the expectational difference equation (1) we substitute future prices forward repeatedly and obtain

$$
P_{t}=\sum_{i=1}^{\infty}\left(\frac{1}{1+r}\right)^{i} \cdot E_{t}\left(D_{t+i}\right)+\lim _{n \rightarrow \infty}\left(\frac{1}{1+r}\right)^{n} \cdot E_{t}\left(P_{t+n}\right) \text {. }
$$

Ruling out speculative bubbles by assuming validity of the transversality condition

$$
\lim _{n \rightarrow \infty}\left(\frac{1}{1+r}\right)^{n} \cdot E_{t}\left(P_{t+n}\right)=0,
$$

we obtain the unique fundamental stock price

$$
P_{t}=P_{t}^{f}=\sum_{i=1}^{\infty}\left(\frac{1}{1+r}\right)^{i} \cdot E_{t}\left(D_{t+i}\right)
$$

The basic idea behind a rational bubble is that there are mathematical expressions $B_{t}$ that are (1) consistent with the limit-term appearing on the right-hand side of Eq. (2), and (2) may satisfy the Euler Eq. (1):

$$
P_{t}=P_{t}^{f}+B_{t}=\sum_{i=1}^{\infty}\left(\frac{1}{1+r}\right)^{i} \cdot E_{t}\left(D_{t+i}\right)+B_{t}
$$

In view of Eq. (5), we interpret the rational bubble $B_{t}$ as the deviation of the current stock price $P_{t}$ from its current fundamental value $P_{t}^{f}$. The entire class of solutions to the Euler Eq. (1) is given by Eq. (5) in which $B_{t}$ is any random variable satisfying the (discounted) martingale property

$$
E_{t}\left(B_{t+1}\right)=(1+r) \cdot B_{t} \quad \text { or, equivalently, } \quad B_{t}=\frac{1}{1+r} \cdot E_{t}\left(B_{t+1}\right) .
$$

The bubble term $B_{t}$ is called rational because its presence in Eq. (5) is consistent with rational expectations. 
To analyze the impact of rational bubbles on stock-price volatility, we follow Evans (1991) who suggests an empirically plausible class of bubbles that are nonlinear, positive, periodically collapsing and satisfy the martingale property (6). Defining the discount factor $\psi=(1+r)^{-1}$, we write the Evans bubble in the form

$$
B_{t}=\left\{\begin{array}{ll}
\frac{1}{\psi} B_{t-1} u_{t}, & \text { if } B_{t-1} \leq \alpha \\
{\left[\theta+\frac{1}{\pi \psi}\left(B_{t-1}-\theta \psi\right) \nu_{t}\right] u_{t},} & \text { if } B_{t-1}>\alpha
\end{array},\right.
$$

where $\theta$ and $\alpha$ are real constants such that $0<\theta<(1+r) \alpha .\left\{u_{t}\right\}_{t=1}^{\infty}$ is an exogenous process of i.i.d. random variables with $u_{t}>0$ and $E_{t-1}\left(u_{t}\right)=1$ for all $t$. As in Evans (1991), we explicitly assume the variables $\left\{u_{t}\right\}$ to be lognormally distributed and scaled to have unit mean, i.e. we assume $u_{t}=\exp \left(y_{t}-\iota^{2} / 2\right)$ with $\left\{y_{t}\right\}_{t=1}^{\infty}$ being i.i.d. $N\left(0, \iota^{2}\right) .\left\{\nu_{t}\right\}_{t=1}^{\infty}$ is an exogenous i.i.d. Bernoulli process independent of $\left\{u_{t}\right\}_{t=1}^{\infty}$ with $\operatorname{Pr}\left(\nu_{t}=1\right)=\pi$ and $\operatorname{Pr}\left(\nu_{t}=0\right)=1-\pi$ for $0<\pi \leq 1$. The event $\left\{\nu_{t}=1\right\}$ means that the bubble will continue to grow, whereas the bubble bursts in case of $\left\{\nu_{t}=0\right\}$.

It is instructive to note that the Evans bubble (7) has two different rates of growth. For $B_{t-1} \leq \alpha$ the bubble grows at the mean rate $\frac{1}{\psi}$. In case of $B_{t-1}>\alpha$ the bubble grows at the faster rate $\frac{1}{\pi \psi}$, but collapses with probability $1-\pi$ per period. When the bubble collapses, it falls back to the mean value $\theta$ and the process recommences.

\section{Conditional stock-price volatility}

In order to compute the conditional volatility associated with the stock-price dynamics given in the Eqs. (5) and (7), it remains to specify a stochastic process $\left\{D_{t}\right\}$ governing the dividend payments. In line with recent literature we assume that dividends follow a driftless random walk of the form

$$
D_{t}=D_{t-1}+\epsilon_{t}
$$

where $\epsilon_{t}$ is an i.i.d. Gaussian white-noise process with mean zero and variance $\sigma^{2}$ (see Al-Anaswah and Wilfling, 2011). Inserting this into Eq. (5), we obtain 


$$
P_{t}=\beta D_{t}+B_{t}=\beta D_{t-1}+B_{t}+\beta \epsilon_{t},
$$

where $\beta=1 / r$.

We now compute the variance of the stock price $P_{t}$ given in Eq. (9) conditional on all information available to market participants as of date $t-1$, which we denote by $\operatorname{Var}_{t-1}\left(P_{t}\right)$. The associated information set $\Omega_{t-1}$ contains, inter alia, all past dividends and stock prices. Additionally, we assume that we can observe (or at least estimate) past values of the bubble component. ${ }^{1}$ From Eq. (9) we have

$$
\operatorname{Var}_{t-1}\left(P_{t}\right)=\operatorname{Var}_{t-1}\left(\beta D_{t-1}+B_{t}+\beta \epsilon_{t}\right)=\operatorname{Var}_{t-1}\left(B_{t}+\beta \epsilon_{t}\right)
$$

Since the dividend error process $\left\{\epsilon_{t}\right\}$ is by definition uncorrelated with the bubble process $\left\{B_{t}\right\}$, Eq. (10) reduces to

$$
\operatorname{Var}_{t-1}\left(P_{t}\right)=\operatorname{Var}_{t-1}\left(B_{t}\right)+\beta^{2} \sigma^{2}
$$

Obviously, when dividends follow a random walk, conditional stock-price volatility is (up to a constant) completely characterized in terms of the conditional variance of the bubble term. The conditional variance $\operatorname{Var}_{t-1}\left(B_{t}\right)$ itself can be derived from the distributional assumptions of the Evans-bubble specification. More precisely, the lognormal distribution of $u_{t}$ from Eq. (7) implies that for $B_{t-1} \leq \alpha$ the conditional variance of the bubble is given by

$$
\operatorname{Var}_{t-1}\left(B_{t}\right)=\left(\frac{1}{\psi} B_{t-1}\right)^{2} \cdot\left[\exp \left(\iota^{2}\right)-1\right] \text {. }
$$

The case $B_{t-1}>\alpha$ is slightly more laborious because it involves the two random variables $u_{t}$ and $\nu_{t}$. In a first step, Eq. (7) allows us to write

$$
\operatorname{Var}_{t-1}\left(B_{t}\right)=\operatorname{Var}_{t-1}\left(\theta u_{t}+\left[\frac{B_{t-1}-\theta \psi}{\pi \psi}\right] \nu_{t} u_{t}\right)
$$

Next, we have to take account of the covariance of the variables $u_{t}$ and $\nu_{t} u_{t}$ in Eq. (13),

\footnotetext{
${ }^{1}$ We will tackle this issue more concretely in Section 4.
} 
which is given by $\pi \cdot\left(\exp \left\{\iota^{2}\right\}-1\right)$. In similar vein, it is straightforward to find $\operatorname{Var}_{t-1}\left(u_{t}\right)$ and $\operatorname{Var}_{t-1}\left(\nu_{t} u_{t}\right)$. Overall, we obtain for $B_{t-1}>\alpha$

$$
\begin{aligned}
\operatorname{Var}_{t-1}\left(B_{t}\right)= & \theta^{2} \cdot \operatorname{Var}_{t-1}\left(u_{t}\right)+\left(\frac{B_{t-1}-\theta \psi}{\pi \psi}\right)^{2} \cdot \operatorname{Var}_{t-1}\left(\nu_{t} u_{t}\right) \\
& +2 \theta\left(\frac{B_{t-1}-\theta \psi}{\pi \psi}\right) \cdot\left[\pi \cdot\left(\exp \left\{\iota^{2}\right\}-1\right)\right] \\
= & \theta^{2}\left(\exp \left\{\iota^{2}\right\}-1\right)+\left(\frac{B_{t-1}-\theta \psi}{\pi \psi}\right)^{2} \cdot\left(\exp \left\{\iota^{2}\right\} \cdot \pi-\pi^{2}\right) \\
& +2 \theta\left(\frac{B_{t-1}-\theta \psi}{\pi \psi}\right) \cdot\left[\pi \cdot\left(\exp \left\{\iota^{2}\right\}-1\right)\right] .
\end{aligned}
$$

Now, inserting Eq. (14) into Eq. (11), the conditional stock-price variance is given by

$$
\operatorname{Var}_{t-1}\left(P_{t}\right)=\left\{\begin{array}{ll}
\left(\frac{1}{\psi} B_{t-1}\right)^{2}\left(\exp \left\{\iota^{2}\right\}-1\right)+\beta^{2} \sigma^{2}, & \text { if } B_{t-1} \leq \alpha \\
& {\left[\theta^{2}+2 \theta\left(\frac{B_{t-1}-\theta \psi}{\psi}\right)\right] \cdot\left(\exp \left\{\iota^{2}\right\}-1\right)} \\
+\left(\frac{B_{t-1}-\theta \psi}{\pi \psi}\right)^{2}\left[\exp \left\{\iota^{2}\right\} \pi-\pi^{2}\right]+\beta^{2} \sigma^{2}, & \text { if } B_{t-1}>\alpha
\end{array} .\right.
$$

\section{Bubble and stock-price volatility}

\subsection{Theoretical results}

To state a first theoretical result we note from Eq. (15) that the bubble term $B_{t-1}$ has an increasing effect on conditional stock-price volatility. This implies that the mere existence of a speculative bubble necessarily increases stock-price volatility. We may analyze this impact further by considering the derivative

$\frac{\partial \operatorname{Var}_{t-1}\left(P_{t}\right)}{\partial B_{t-1}}=\left\{\begin{array}{ll}\frac{2}{\psi^{2}} B_{t-1} \cdot\left(\exp \left\{\iota^{2}\right\}-1\right), & \text { if } B_{t-1} \leq \alpha \\ \frac{2}{\psi^{2}}\left[\left(B_{t-1}-\theta \psi\right) \cdot\left(\frac{\exp \left\{\iota^{2}\right\}}{\pi}-1\right)+\theta \psi\left(\exp \left\{\iota^{2}\right\}-1\right)\right], & \text { if } B_{t-1}>\alpha\end{array}\right.$.

Eq. (16) establishes a strictly positive relationship between the infinitesimal change in stock-price volatility and $B_{t-1}$, the bubble level from the previous period. Consequently, an explosive bubble path necessarily entails an explosive path of stock-price 
volatility.

Next, we address the impact of a bubble burst on stock-price volatility. Since the conditional stock-price variance given in Eq. (15) is a function of $B_{t-1}$, stockprice volatility collapses one period after the bubble burst. Furthermore, owing to the Eqs. (15) and (16) the stock-price volatility process attains its maximum when $B_{t-1}$ takes on its largest value which typically occurs on the eve of the bubble crash. This theoretical result is consistent with the volatility dynamics described by Brunnermeier and Oehmke (2013) and the Minsky model.

\section{Figure 1 about here}

\subsection{Empirical application}

Figure 1 gives an example of an Evans bubble process (right panel) and the stockprice process from the Eqs. (7) and (9) with one large and one moderate bubble. The dividends from Eq. (8) follow a random walk with standard deviation $\sigma=0.03$. We set the parameter value $\beta=50$ while the parameters relevant to the simulation of the Evans bubble are chosen as $\psi=0.9804, \iota^{2}=0.001, \theta=1.1, \pi=0.98$ and $\alpha=2$. Our artificial dataset consists of 250 observations corresponding to a time span of approximately 21 years on the basis of monthly data.

An important stipulation inherent in our theoretical framework concerns the structure of the information set $\Omega_{t-1}$. We explicitly assume that $\Omega_{t-1}$ contains the bubble time series, which is crucial to analyzing stock-price volatility. However, in practice bubble values are unobservable so that we are forced to estimate the bubble process from the data.

To this end, we use a sequential Monte-Carlo method - the so-called particle filteras introduced by Gordon et al. (1993). This Bayesian approach enables us to estimate a latent variable (our bubble process) from nonlinear and non-Gaussian state-space models. In a first step we transform the Eqs. (7) and (9) into a nonlinear state-space form from which we then estimate-besides all other model parameters - the unobservable bubble process. Several technical approaches to parameter estimation via the 
particle filter are available in the literature (see Doucet et al. (2001) for an overview). In this paper we apply the Expectation Maximization (EM) algorithm as proposed by Schön et al. (2011). Additionally, we use a stable estimator of the information matrix established by Duan and Fulop (2011) to compute standard errors. ${ }^{2}$

\section{Table 1 about here}

Table 1 displays the estimates of the parameters from the Eqs. (7) and (9). Owing to an identification problem, we set the parameter $\alpha=2$. The variance of the error term in Eq. (9) equals $\beta^{2} \times \sigma^{2}$ implying the estimate $\hat{\sigma}=0.0321$ of the dividend standard deviation in Eq. (8).

Figure 2 about here

Besides parameter estimation the particle filtering approach allows us to estimate the bubble process from the data. Figure 2 displays the estimated bubble process (bold line) as well as the true (simulated) bubble process (taken from the right panel in Figure 1). Obviously, the estimated bubble process almost perfectly fits the true bubble values. These econometrically reliable estimates of the bubble process can now be included in the information set $\Omega$. This procedure ultimately enables us to analyze the conditional stock-price variance according to Eq. (15).

Figure 3 about here

Figure 3 displays the conditional stock-price variance (bold line) along with the estimated bubble process (thin line). Two features are worth mentioning. (1) In line with our theoretical results from above, stock-price volatility collapses one period after the bubble burst. (2) The increase in the stock-price variance process in response to increases in the bubble process occurs with a considerable time delay. In Figure 3, for example, the bubble process begins to take on substantially increasing values around

\footnotetext{
${ }^{2}$ Technical details of our estimation procedure are available upon request.
} 
the date $t=50$. By contrast, stock-price volatility remains (roughly) constant and begins to increase steadily no earlier than around the date $t=110$.

To explain this latter phenomenon we again refer to the derivative in Eq. (16). For $B_{t-1}>\alpha$ the slope of the stock-price variance essentially consists of the two summands within the squared brackets. For small and moderate values of $B_{t-1}$ the first term is negligible and the slope of the stock-price variance is primarily determined by the constant value $\frac{2 \theta}{\psi}\left(\exp \left\{\iota^{2}\right\}-1\right)$. During this period the stock-price variance remains largely unaffected by the $B_{t-1}$-levels. It is not until the bubble values get sufficiently large that the first summand (containing $B_{t-1}$ ) begins to dominate the slope of the stock-price variance triggering strongly increasing stock-price volatility.

The stock-price volatility dynamics displayed in Figure 3 is strongly consistent with the observations by Brunnermeier and Oehmke (2013) on the distinct volatility phases in the run-up to a financial crisis. If we interpret the start of the crisis as the first period after the burst of the stock-market bubble, their observation can be stated as follows. In an early stage when the bubble begins to emerge, stock market volatility is comparably low. Then, volatility increases due to trading frenzy in a phase of euphoria which finally ends in the burst of the bubble. At the beginning of the financial crisis, that is one period after the crash, stock-market volatility is maximal.

\section{Conclusion}

This paper analyzes (conditional) stock-price volatility dynamics in a present-value framework with periodically collapsing bubbles as proposed by Evans (1991). We derive closed-form expressions for the volatility paths and explore their properties theoretically. In an empirical part we describe a sequential Monte Carlo approach for extracting the unobservable bubble process from the data.

Our major finding is that the present-value framework produces stock-price volatility paths that are broadly consistent with empirically observed volatility structures in the run-up to financial crises and/or the burst of a stock-market bubble. Evans bubbles contribute to excessive stock-price volatility and volatility reaches its maximum when 
the bubble bursts. Our volatility results should be of interest to traders in international stock and derivative markets, for example for valuing stock-price sensitive claims.

\section{References}

Al-Anaswah, N., Wilfling, B., 2011. Identification of speculative bubbles using statespace models with Markov-switching. Journal of Banking and Finance 35, 10731086.

Blanchard, O.J., Watson, M.W., 1982. Bubbles, rational expectations and financial markets, In Wachtel, P. (Ed.), Crises in the Economic and Financial System. Lexington Books, Lexington, pp. 295-315.

Brunnermeier, M.K., Oehmke, M., 2013. Bubbles, financial crises, and systematic risk. Handbook of the Economics of Finance 2, 1221-1288.

Campbell, J.Y., Lo, W., MacKinlay, A.C., 1997. The Economdetrics of Financial Markets. Princeton University Press, Princeton.

Cuthbertson, K., Nitzsche, D., 2004. Quantitative Financial Economics: Stocks, Bonds and Foreign Exchange. Wiley, New York.

Doucet, A., de Freitas, N., Gordon, N.J., 2001. Sequential Monte Carlo Methods in Practice. Springer Verlag. New York.

Duan, J.C., Fulop, A., 2011. A stable estimator of the information matrix under EM for dependent data. Statistics and Computing 21(1), 83-91.

Evans, G.W., 1991. Pitfalls in testing for explosive bubbles in asset prices. American Economic Review 81, 922-930.

Flood, R.P., Hodrick, R.J., 1986. Asset price volatility, bubbles, and process switching. Journal of Finance 41(4), 831-842.

Gordon, N.J., Salmond, D.J., Smith, A.F.M., 1993. A novel approach to nonlinear and non-Gaussian Bayesian state estimation. IEE Proceedings. Part F: Radar and Sonar Navigation 140, 107-113.

Kindleberger, C.P., Aliber, R.Z., 2005. Manias, Panics, and Crashes: A History of Financial Crises. John Wiley and Sons, Hoboken. 
Schön, T.B., Wills, A., Ninness, B., 2011. System identification of nonlinear state-space models. Automatica 47, 39-49.

\section{Tables and Figures}

Table 1. Parameter estimates using the particle filter

\begin{tabular}{lccc}
\hline Parameter & True value & Estimate & Standard error \\
\hline$\beta$ & 50.0000 & 50.9366 & 0.0963 \\
$\beta^{2} \times \sigma^{2}$ & 2.2500 & 2.6657 & 0.0868 \\
$\psi$ & 0.9804 & 0.9666 & $3.9591 \times 10^{-5}$ \\
$\iota^{2}$ & 0.0010 & 0.0011 & $2.2510 \times 10^{-12}$ \\
$\theta$ & 1.1000 & 0.5749 & $2.4932 \times 10^{-4}$ \\
$\pi$ & 0.9800 & 0.9925 & $2.4932 \times 10^{-5}$ \\
\hline
\end{tabular}

Simulated stock-price process

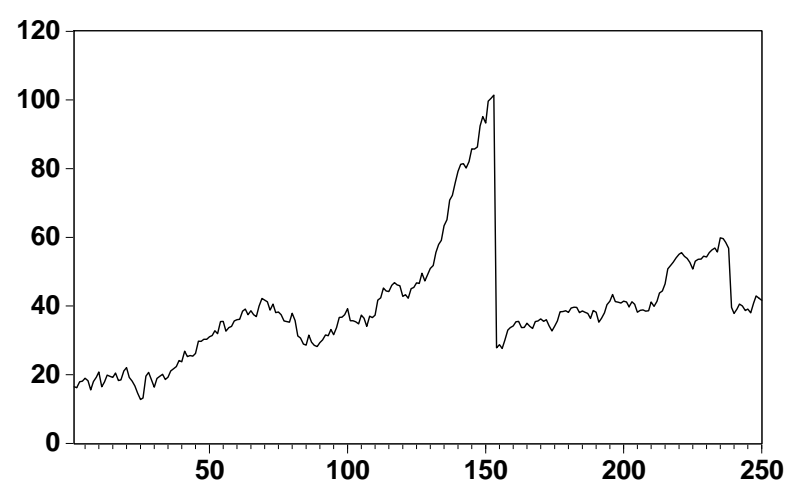

Simulated bubble process

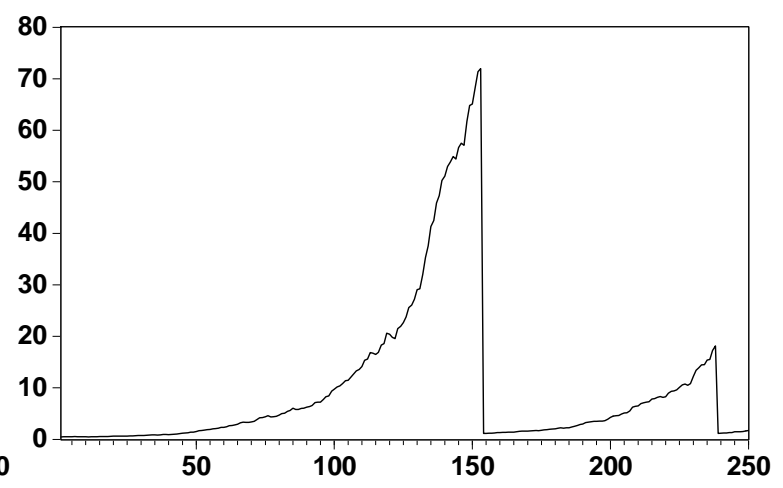

Figure 1. Stock-price process and the included Evans bubble 


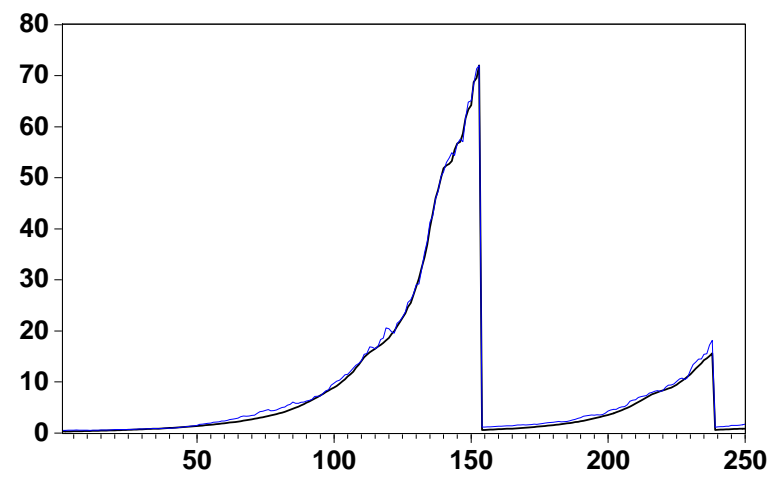

Figure 2. Estimated bubble process (bold line) versus true bubble process (thin line)

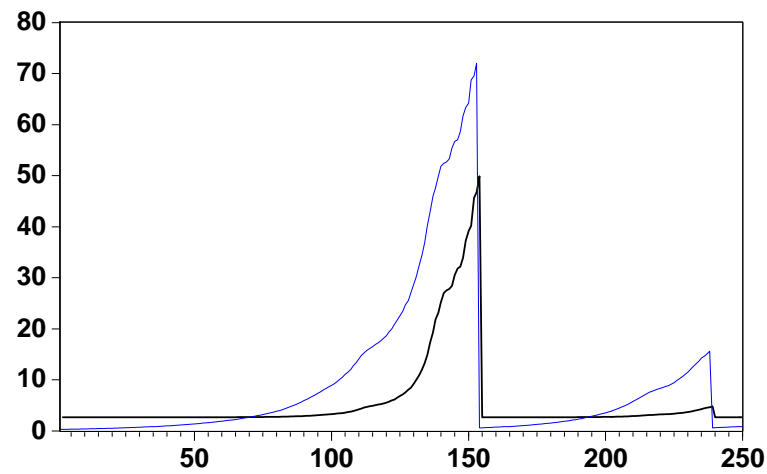

Figure 3. Conditional stock-price variance (bold line) and estimated bubble process (thin line) 